\title{
LA RESPONSABILIDAD SOGIAL EN LA SANIDAD PÚBLICA. UN PLAN INNOVADOR DE VOLUNTARIADO SOLIDARIO EN LOS HOSPITALES
}

\section{SOCIAL RESPONSABILITY IN THE PUBLIC HEALTH SYSTEM. AN INNOUATIVE PLAN FOR SUPPORT AND VOLUNTEERING IN HOSPITALS}

\section{RED SANITARIA DE RSC Y GRUPO UCM DE INNOVA-DOCENCIA 176}

Facultad de Ciencias de la Información. Universidad Complutense.

Avenida Complutense, S/N - E-28040 Madrid, Spain

TIfn: + 0034913942216

Email: nieto@ccinf.ucm.es \& fcabezuelo@ucm.es

PALABRAS CLAVES

Responsabilidad social corporativa, hospitales, España, comunicación
KEY WORDS

Corporate Social Responsability, hospitals, Spain, communications. 


\section{Resumen}

La red del sistema sanitario público es básica para el desarrollo diario de una ciudad que pretende avanzar en el progreso y bienestar de sus ciudadanos y ciudades. Este sistema incluye a todas las organizaciones e instituciones que, con recursos públicos, se ocupan de mantener, promover y restaurar la salud y el bienestar de todos sus habitantes. Sin embargo, cada vez se hace más necesaria una nueva orientación en la prestación de los servicios sanitarios centrada en la atención integral al paciente como persona y como miembro de una comunidad. Este principio rector viene avalado en el caso de España por un grupo de gestores y profesionales de un total de dieciocho hospitales públicos, repartidos en ciudades de cinco Comunidades Autónomas españolas (Aragón, Cataluña, Galicia, Islas Baleares y Madrid), quienes, sensibilizados con la necesidad de aplicar planes de responsabilidad social corporativa (RSC) en sus centros sanitarios, han diseñado un plan estratégico en esta materia dirigido a pacientes con dificultades de atención a domicilio o con carencias de apoyo familiar. Este plan de RSC parte de una investigación socio-sanitaria basada en el análisis de cerca de treinta mil intervenciones sociales llevadas a cabo en seis hospitales públicos españoles. De las conclusiones de este estudio, se deriva que una tercera parte de los pacientes atendidos por los equipos de trabajo social en estos centros, carecía del apoyo necesario para la correcta atención en sus domicilios, lo que prolongaba, no sin riesgos, su estancia hospitalaria.

\section{Abstract}

The Spanish public health system network is basic for the daily development of a city that aims to advance the progress and welfare of its citizens and cities. It includes all organizations and institutions that, with public resources, are responsible for maintaining, promoting and restoring the health and well-being of all its inhabitants. However, a new orientation in the provision of health services is becoming increasingly necessary, focusing on comprehensive patient care as a person and as a member of a community. This guiding principle is endorsed in the case of Spain by a group of managers and professionals from a total of eighteen public hospitals, distributed in cities of five Spanish Autonomous Communities (Aragon, Catalonia, Galicia, Balearic Islands and Madrid), who, sensitized with the need to apply corporate social responsibility (CSR) plans in their health centers, have designed a strategic plan in this area aimed at patients with home care difficulties or with lack of family support. This CSR plan is based on a socio-sanitary research based on the analysis of nearly thirty thousand social interventions carried out in six Spanish public hospitals. From the conclusions of this study, it is derived that a third of the patients attended by social work teams in these centers, lacked the necessary support for proper care in their homes, which prolonged, not without risk, their hospital stay. 


\section{Introducción y marco teórico}

En el actual contexto digital que sufren las ciencias de la información, la búsqueda de la excelencia es una exigencia. Así que de acuerdo con la teoría de la excelencia en la gestión de la comunicación estratégica y las relaciones con los públicos este trabajo toma como fundamento una investigación emprendida por la International Association of Business Communicators (IABC) acerca de las mejores prácticas en la gestión de la comunicación de las organizaciones, durante un periodo total de quince años (J. E. Grunig 1992; Dozier et al. 1995; L. A. Grunig et al. 2002). Esta teoría de excelencia en la gestión de la comunicación con los públicos que integran una organización incluye, además, las aportaciones científicas de la teoría de la gestión estratégica de las relaciones con los públicos, la teoría situacional de los públicos, la teoría de los modelos de las relaciones públicas, la evaluación de las relaciones públicas, la comunicación con empleados y el planteamiento global de las relaciones con los públicos, incorporando a la gestión la consideración de cuestiones tales como el género, la diversidad, el activismo, la ética y la responsabilidad social en las sociedades contemporáneas.

La teoría de la excelencia explica el valor de las relaciones públicas en las organizaciones fundamentado en la responsabilidad social de las decisiones gerenciales y en las relaciones de calidad con los públicos interesados. Según esta teoría, para que una organización sea eficaz debe comportarse de manera que le permita resolver los problemas y satisfacer los intereses de los públicos, a la vez que se alcanzan los objetivos de las organizaciones y comunidades.

Una organización que pretenda comportarse de modo socialmente aceptable debe explorar su entorno para identificar los públicos afectados por sus decisiones gerenciales, y tratar de resolver los problemas que estos públicos consideran importantes para sus ciudadanos..

Para maximizar el valor de las relaciones públicas excelentes es condición necesaria y fundamental la participación en la gestión estratégica de la organización. No es posible emprender un plan integral de excelencia en las relaciones con los públicos sin el compromiso de la alta dirección y el acuerdo de los principales responsables de la organización, entendidos como los líderes de "the dominant coalition" (J. E. Grunig, 2006) o "coalición dominante" en su equivalente en español, aunque sea un término menos conocido en lengua española.

Allá por el año 1998 se demostró ya que la teoría de las relaciones públicas excelentes es aplicable a cualquier contexto, incluyendo la ética como una variable indispensable en las relaciones con los públicos (L. A. Grunig, 1998). En cualquier caso, la tendencia en la práctica de las relaciones públicas excelentes parece orientarse hacia estrategias globales y, a la vez, hacia la especialización por áreas de actividad (Toth 2007). Este es precisamente el caso que presentamos, un ejemplo de aplicación de las relaciones públicas de excelencia en los hospitales y áreas de salud del sistema sanitario público español. De todo lo anterior se deriva que las relaciones públicas de excelencia sólo pueden plantearse desde la responsabilidad social corporativa en las actuales sociedades occidentales. 
Como señala García Nieto (2012), la responsabilidad social corporativa es arrogada por una organización cuando ésta responde a la necesidad de asumir las consecuencias de su gestión, de sus políticas, de sus procedimientos y de sus conductas, en relación con los diferentes grupos sociales, contemporáneos o futuros, afectados por ella a corto, medio o largo plazo. Incorporar la responsabilidad social en la genética empresarial sólo es posible, si la empresa trasciende su naturaleza económica y se erige como una entidad social en continuo proceso de interacción y adaptación con su entorno (García Nieto, 2012: 94).

Con esta premisa y centrándonos exclusivamente en la perspectiva de las relaciones públicas, encontramos tres planteamientos diferentes de interés científico sobre la responsabilidad social corporativa (García Nieto, 2012: 101) en las sociedades contemporáneas. La teoría de los stakeholders de Freeman (Freeman, 1983; Freeman y Gilbert, 1988; Freeman, Wicks y Parmar, 2004), la teoría de la pirámide de la responsabilidad social corporativa de Carroll (Carroll, 1991, 1999; Carroll y Buchholtz, 2011), y la propuesta de los niveles de RSC dentro de la teoría de los modelos de las relaciones públicas de James E. Grunig (Grunig \& Hunt, 1984 y Grunig, 1992).

En esta categoría de responsabilidad social (Grunig), ética y filantrópica (Carroll) y solidaria (García Nieto) se integran los planes y acciones que las organizaciones emprenden con el fin de garantizar el bienestar social y la mejora de la calidad de vida de las personas. Es este nivel de responsabilidad solidaria en el que, precisamente, se circunscribe el plan de responsabilidad social corporativa que proponemos en el ámbito de los hospitales públicos de los que disfruta la ciudadanía en España.

\section{Objetivos}

En la actualidad, los servicios médicos a la ciudadanía deben concebirse como una actividad al servicio de todos motivada por el beneficio del paciente. Los fines de la medicina, de acuerdo con el grupo de estudios de The Hastings Center de Nueva York, prestigioso centro de investigación en bioética, deben ser algo más que la curación de la enfermedad y el alargamiento de la vida. Es preciso atender, además, aspectos tan sustanciales como la prevención de las enfermedades o la paliación del dolor y el sufrimiento. Curar y cuidar implican el mismo nivel de relevancia (Fundació Víctor Grífols i Lucas, 2005).

Así lo piensan, también, un grupo de gestores de seis hospitales públicos españoles y las áreas de salud de cinco Comunidades Autónomas españolas (Aragón, Cataluña, Galicia, Islas Baleares y Madrid), que, con la iniciativa de innovar en la gestión sanitaria en la búsqueda de la excelencia, deciden poner en marcha un proyecto de responsabilidad social corporativa (RSC a partir de ahora). Se trata de un plan que se emprende desde la gestión de la comunicación y las relaciones públicas con resultados equilibrados, priorizando la satisfacción de los intereses públicos, pero siendo conscientes de las consecuencias positivas que este plan provocará en la imagen pública y la aceptación social de los centros sanitarios.

Este trabajo parte de un contexto y equipo de trabajo muy concreto, como se explica a continuación. El 
plan de responsabilidad social corporativa que se presenta, pretende ser un modelo de referencia para el resto de los hospitales de la red pública en España. En este caso, la parte del diseño de la comunicación desde la propuesta universitaria corre a cargo del Proyecto Innova-Docencia $N^{\circ}$ de referencia 176, 2017/2018, titulado "Universidad y sociedad: comunicación e integración en empresas e instituciones públicas y organizaciones no lucrativas. Nuevos avances" de la Universidad Complutense de Madrid (UCM), dirigido por la profesora Dra. María Teresa García Nieto, y que cuenta con la participación de un grupo de profesoras, profesores y colaboradoras de la UCM y de la Universidad CEU San Pablo (USP-CEU), así como de un grupo de estudiantes pertenecientes a diferentes programas de doctorado y máster.

Por su parte, la Red Sanitaria de RSC está coordinada por la doctora Ana María Díaz-Oliver (Hospital 12 de Octubre de Madrid) y compuesta por los siguientes responsables de la gestión de hospitales públicos y áreas de salud: Antonio Jesús Alemany López (Subgerente del Hospital Clínico de San Carlos de Madrid), Juan Carlos Alonso Punter (Director de Económico Financiero de la Hospital Universitario Fundación Alcorcón), Pilar Ayala (Directora del Instituto del Hospital Clínic de Barcelona), Anna Borruel (Jefa de Atención al Usuario y trabajo social del Hospital Joan XXIII de Tarragona), Luz Campello García (Responsable de Trabajo Social del Hospital A Coruña), Lourdes Castellano Del Pozo (Trabajadora Social del Hospital 12 de Octubre de Madrid), Ana María Díaz-Oliver Fernández-Hijicos (Jefa de Servicio de Responsabilidad Social Corporativa del Hospital 12 de Octubre), Javier Fernández Nistal (Director de Recursos Humanos del Hospital A Coruña), Maribel Iloro (Directora de Humanización Hospital Clínico San Carlos), Pedro Luis Izquierdo Doyagüez (Director de Gestión del Hospital Clínico de San Carlos), Laura Larriba Leira, (Responsable de Calidad Ambiental del Hospital A Coruña), Luis Manuel Maduell Folch (Director de Compras del Hospital Juan XXIII), María Mateu (Jefa de Responsabilidad Social Corporativa del Hospital Clínic de Barcelona), Gemma Pastor García (Responsable de Medio Ambiente del Hospital Clínico San Carlos), Carmen Sánchez Roldan (Subdirectora de Gestión de Participación y Ciudadanía de Aragón), Pablo Serrano Balazote (Director de Planificación del Hospital Universitario 12 de Octubre), Ángela María Tumbarello Casciola (Jefe de Unidad Responsabilidad Social Corporativa del Servicio de Salud de las Islas Baleares) y Manuela Vázquez (Trabajadora Social del Hospital Joan XXIII).

\section{Metodología}

De acuerdo con los fundamentos de la gestión de la comunicación y de las relaciones públicas, todo plan estratégico de responsabilidad social corporativa requiere, como ya se ha indicado, de una definición certera de la realidad en la cual nos encontramos. Por ello, el primer paso debe ser, necesariamente, la recogida de información suficiente, válida y fiable que permita plantear unos objetivos realistas y tomar las decisiones adecuadas, en cuanto a estrategias y acciones, para poder alcanzar las metas con éxito.

Para ello, se ha contado con la siguiente metodología. Para la recogida de la necesaria información hemos recurrido a las siguientes fuentes. Entre las fuentes primarias, el primer paso para la planificación 
de este modelo de RSC es el estudio específico de las necesidades sociales que se detectan en el proceso de ingreso hospitalario de los pacientes. Concretamente se identifican y se evalúan las carencias de las personas que se encuentran en situación de un nivel moderado de dependencia.

Los hospitales seleccionados para la realización de este estudio no constituyen una muestra aleatoria, sino condicionada. Es, precisamente, la detección pionera de un rasgo de sensibilidad y predisposición favorable de sus gestores hacia la puesta en marcha de planes de actuación socialmente responsables, lo que determinó el planteamiento de este proyecto. Así, pues, para la ejecución del estudio de las necesidades sociales se han recogido y analizado los expedientes de las problemáticas sociales de los siguientes hospitales, todos ellos pertenecientes a la red de hospitales públicos de las Comunidades Autónomas de Galicia, Cataluña y Madrid, y con una población total de referencia de dos millones de usuarios: Hospital Universitario da Coruña, Hospital Universitario de Santiago de Compostela, Hospital Universitario de Tarragona Joan XXIII, Hospital Virgen de la Cinta de Tortosa, Hospital Clínico San Carlos y Hospital Universitario 12 de Octubre (12-O) de Madrid.

Se han utilizado las bases de datos de las unidades de trabajo social de todos los hospitales mencionados, seleccionando aquellos registros que incluyeran temas relacionados con problemáticas sociales.

De las bases de datos nutridas a partir de las aportaciones recogidas por los trabajadores sociales de los Hospitales se han obtenido los casos de estudio relativos a pacientes que han sido objeto de intervención por parte de dichos profesionales. Para la selección de los casos, los pacientes de referencia debían reunir las dos siguientes condiciones. Por un lado, pacientes atendidos en los hospitales mencionados desde el 2009 hasta 2014 (tanto en hospitalización como en consultas por algún proceso clínico). Y por otro lado, pacientes derivados al servicio de Trabajo Social desde el área asistencial, por petición del propio paciente o por otro tipo de instituciones (Atención Primaria, Servicios Sociales, Penitenciarías).

Además, se han explotado los sistemas informáticos de los Hospitales que participan en el presente proyecto. En concreto: Hp-Doctor del Hospital Universitario 12 de Octubre, Rusho del Hospital Clínico San Carlos, Siuac del Hospital de Tarragona, Social del Hospital de Santiago de Compostela y Asís del Hospital da Coruña.

Finalmente, respecto a las fuentes secundarias, se ha realizado una búsqueda documental en diferentes catálogos y bases de datos. Algunas de carácter multidisciplinar, como la Academic Search Complete, y otras específicas, como Medline, Psyclnfo, Socindex, Social Work Abstract, Family \& Society Study World Wide, Psychology and The Behavior Sciences Collection.

La búsqueda de esta literatura científica se ha centrado en el período de los últimos diez años y las palabras clave utilizadas como referencia para la selección en el proceso de búsqueda han sido: "volunteer", "volunteering", "hospitalization" y "home-based care", teniendo en cuenta las características del plan de RSC que se pretende planificar. La elección de las palabras clave vino determinada por los primeros resultados de la investigación con las fuentes primarias. 


\section{Resultados de la investigación y la identificación del problema social}

Sin duda alguna, en las actuales sociedades contemporáneas, el cambio en la estructura social conlleva repercusiones en la gestión hospitalaria, ya que los cuidados a enfermos con incapacidad para valerse por sí mismos se han exigido cambios en las últimas décadas. Han pasado de ser asumidos tradicionalmente por las familias a ser demandados como una ayuda proporcionada por la propia administración o por otros diferentes colectivos. A ello se suma el progresivo envejecimiento de la población española que incrementa la necesidad de una atención especial, con un abordaje multidisciplinar a la cronicidad, mediante planes de atención específicos.

La población mundial está envejeciendo. De 575.000 millones de ancianos en 1995 (entendiendo por anciano persona mayor de 65 años) se pasará a 1.200 .000 millones en el 2025, según estudios realizados por Murphy et al. (2009). Carabaña (2003) señala que esta situación se agrava considerablemente en España, debido, sobre todo, a los bajos índices de natalidad situados por debajo de la media de Europa y del mundo. La crisis y el paro se apuntan como las variables que más han contribuido al aumento de la población envejecida. En el cuadro que se expone a continuación se recoge la estimación de la evolución de la población mayor de 65 y de 85 años desde 1900 hasta el 2050, según el Instituto Nacional de Estadística (INE).

De este modo, el incremento de las atenciones sanitarias implica un aumento de demandas sociales. Como señala Fernando Lamata (Fundadeps, 2011), el paradigma de la sanidad en el siglo XXI ha cambiado. Ya no se trata sólo de curar o sanar, sino de cuidar, un concepto más responsable. Dar cuidados implica ocuparse del cuerpo y de la mente, buscando mayores índices de felicidad para todos los que se relacionan con un hospital. Dar cuidados, desde esa concepción, pasa, también, por facilitar el retorno del paciente a su ámbito social, a su domicilio, lo más rápidamente posible y en las mejores condiciones de salud. Así, la visión de la organización hospitalaria de un mundo más saludable, un mundo mejor, tiene que complementarse con unos valores reflejados en la responsabilidad social corporativa respetando la intimidad, la integridad del paciente y el medio ambiente; además de tener en cuenta las expectativas de los ciudadanos.

La calidad y la seguridad en el servicio sanitario son preocupaciones comunes a todos los servicios de salud y representan un ámbito estratégico de cualquier organización sanitaria. El enfoque de los proyectos de calidad, a menudo, prioriza los resultados de una buena gestión del proceso asistencial de una organización, sobre los resultados de una adecuada atención integral y personalizada. Ello podría explicar por qué el aumento del gasto sanitario en períodos previos no ha ido necesariamente ligado a un aumento de la satisfacción de los usuarios.

En la información recogida en los seis hospitales de nivel 3 (es decir, grandes hospitales son aquellos que cuentan la mayoría de las especialidades) que participan en el presente proyecto, se observa sus Unidades de Trabajo Social han realizado 29.182 intervenciones sociales anuales. Del total de estas intervenciones, aproximadamente un $33 \%$ corresponde a problemáticas sociales relacionadas con la dificultad de atención a 
domicilio por insuficiencia o carencia de apoyo familiar. Los cuadros de mando de dichos hospitales ratifican el dato señalado. A continuación, en la siguiente tabla se muestran las intervenciones de Trabajo Social en altas hospitalarias:

\begin{tabular}{|l|l|l|l|l|l|}
\hline $\begin{array}{l}\text { MUESTRA DE HOSPITALES } \\
\text { PÚBLICOS }\end{array}$ & Altas hospitalarias & $\begin{array}{l}\text { Intervenciones de } \\
\text { Trabajo Social } \\
\text { (ITS) }\end{array}$ & \% ITS/Altas & $\begin{array}{l}\text { ITS por falta de } \\
\text { apoyo familiar }\end{array}$ & $\begin{array}{l}\text { \% ITS por falta de } \\
\text { apoyo sobre altas }\end{array}$ \\
\hline Hospital 12-O & 42.428 & 13.670 & $32,22 \%$ & 4.511 & $10,63 \%$ \\
\hline Hospital Clínico & 33.309 & 5.803 & $17,42 \%$ & 1.915 & $5,75 \%$ \\
\hline Hospital de Tarragona & 20.021 & 1.426 & $7,12 \%$ & 471 & $2,35 \%$ \\
\hline Hospital de Tortosa & 11.126 & 1.141 & $10,26 \%$ & 377 & $3,38 \%$ \\
\hline Hospital da Coruña & 40.892 & 5.084 & $12,43 \%$ & 1.678 & $4,10 \%$ \\
\hline Hospital de Santiago & 37.230 & 2.058 & $5,53 \%$ & 679 & $1,82 \%$ \\
\hline TOTAL & 185.006 & 29.182 & $15,77 \%$ & 9.630 & $5,21 \%$ \\
\hline
\end{tabular}

Tabla 1. Intervenciones de Trabajo Social en relación con las Altas de Hospitalización (Fuente: elaboración propia Red Sanitaria de RSC).

En ese contexto, la realidad de la hospitalización actual, en la que se procura la derivación de los pacientes a los ambulatorios de atención primaria y la reducción de las estancias en los hospitales, parece aconsejar la dinamización de los procesos. Se pretende minorar las estancias hospitalarias y fomentar alternativas al ingreso convencional que mejoren la calidad de vida de los pacientes y eviten las consecuencias no deseables de los ingresos prolongados (por ejemplo: infecciones nosocomiales). Tal y como afirman Mendoza, Navarro, Sánchez-Quijano, Villegas, Asencio y Lissen, (2012) las estancias prologadas por motivos no médicos son frecuentes y principalmente motivadas por el problema social familiar para hacerse cargo de los pacientes después de su hospitalización. Esto representa una sobrecarga importante para los hospitales, no solo desde el punto de vista económico sino, también, desde la eficiencia social y, en consecuencia, desde la calidad percibida prestada al paciente.

En algunos hospitales, para favorecer ese proceso, se han creado unidades de medicina hospitalaria. Estas unidades desarrollan un programa de atención especial al paciente frágil y dependiente que pivota fundamentalmente en la enfermera gestora de enlace hospitalario, quien realiza una minuciosa valoración de los pacientes y establece sus necesidades en cuidados y recursos. De esta manera, se facilita el alta a su domicilio o el traslado a otra de las nuevas estructuras facilitadoras del proceso asistencial: la hospitalización a domicilio $(\mathrm{HaD})$.

Para que un paciente sea incluido en el programa de HaD debe cumplir los siguientes requisitos: 
aceptación por parte del paciente, tener un cuidador válido presencial, aceptación del cuidador, estabilidad clínica y diagnóstico claro, necesidad de cuidados y/o tratamiento de rango hospitalario, comunicación telefónica, isócrona de 30 minutos y/o pertenencia al área sanitaria del centro hospitalario.

En cualquiera de los dos casos, alta a domicilio o traslado a $\mathrm{HaD}$, es necesaria la presencia de un cuidador. Cuando un paciente es trasladado a su domicilio con $\mathrm{HaD}$, el cuidador se convierte en el garante de la prestación del servicio de HaD pues participa en la comunicación, en el aseguramiento de los cuidados básicos (alimentación, higiene, toma de medicación oral, aerosolterapia...), en el acompañamiento a las pruebas hospitalarias cuando sea necesario... Se convierte en la persona clave, nexo entre el paciente y el hospital.

En este contexto, la intervención de la enfermera gestora de enlace hospitalario es un apoyo necesario en los procesos hospitalarios, pero, también, es imprescindible desarrollar programas de apoyo al paciente y a los cuidadores en el domicilio. Programas que faciliten la estancia del paciente en el hogar familiar y evite los traslados innecesarios a los servicios de urgencia, por problemas que pueden manejarse en el domicilio con la adecuada educación sanitaria y soporte social.

Por ello, la transición del hospital al domicilio es un momento de vulnerabilidad para todos los pacientes y sobre todo para los mayores de 65 años. Si no se manejan adecuadamente tienen el riesgo de precisar la readmisión en el hospital. Ello puede contribuir a disminuir la calidad de vida y a incrementar el peligro de su estado de salud. Una revisión actual de la literatura en relación con el problema de los reingresos de la población anciana, realizada por L.M. Wolfe (2013), muestra que hay un número limitado de los recursos disponibles para los hospitales.

Centrándonos en el procedimiento de atención sanitaria de cualquier ciudadano, podríamos afirmar que a partir de un problema clínico que implique un ingreso, la persona comienza a ser "paciente" y se desencadenan acciones que repercuten en su vida diaria, entorno familiar y social. Por ello, son ámbitos interrelacionados y vinculados. Estudios realizados en Estados Unidos (Rydeman, Tornkwist, Agreus, Dahlberg, 2012) señalan que las personas mayores y sus familiares se sienten perdidos en el contexto hospitalario. Cuando dicho colectivo pierde su autonomía se sienten indefensos. Muchas veces se encuentran en medio de las discusiones entre los profesionales del Sistema Sanitario de Salud y los familiares.

El mundo del paciente es el mundo de partida del trabajo científico. Se pueden considerar las mismas circunstancias para dos pacientes diferentes $\mathrm{y}$, sin embargo, la experiencia y las expectativas de cada uno de ellos son distintas. Por tanto, es conveniente aumentar los esfuerzos para dar recursos a las personas mayores y sus familiares con el fin de que continúen con la misma vida en sus domicilios. Rydeman et al. (2012) afirman, fruto de su investigación, que hay que crear un puente entre la vida hospitalaria y el domicilio del paciente.

En esa línea, el voluntariado ocupa un papel clave para hacer que ese puente sea más humano; puede 
contribuir a amortiguar el cambio drástico que viven los pacientes tras su paso por el Hospital. Además, los voluntarios viven la experiencia positivamente. Un ejemplo de ello es el testimonio de Darlene Andrews miembro del Spirit of Cobb Charter Chapter en Marietta, Georgia (EEUU), tras colaborar con pacientes en cuidados paliativos afirmaba que era una experiencia muy gratificante que le había enseñado "la paciencia, la tolerancia y más empatía hacia las personas que ya no pueden controlar sus vidas". Incluso la experiencia de ser voluntario puede provocar vocación de cuidados y generar un incremento de profesionales dedicados a la Sanidad, Advice P.R.N. (2005).

En definitiva, y a modo de resumen, podemos afirmar que el plan de RSC que proponemos, centrado en la creación de una plataforma de acompañamiento con voluntariado, es esperado y necesario, fundamentalmente por las siguientes circunstancias:

a) El incremento del envejecimiento en la población española. En algunas especialidades como Traumatología tiene una media de edad de 81 años.

b) La duplicidad del número de actos asistenciales prolonga las estancias considerablemente.

c) El aumento del número de demandas sociales que se solicitan en las unidades de trabajo social, especialmente cuando las limitaciones sociales están relacionadas con la dificultad de atención a domicilio por carencia de apoyo familiar.

En virtud de todo ello, la sociedad española se enfrenta a una realidad caracterizada por una elevada población envejecida, cada vez más demandante de actos asistenciales, tanto en las consultas, como en las cirugías, a lo que habría que añadir la consecuente prolongación de las estancias hospitalarias. En esta situación, nuestro proyecto se presenta como una posible solución para paliar una previsible sobrecarga para el Sistema Sanitario, así como el posible deterioro de la percepción de la calidad en la atención.

\section{El plan de responsabilidad social corporativa}

El plan de responsabilidad social corporativa que planteamos está encaminado a crear un modelo o plataforma de acompañamiento, mediante voluntariado social, a pacientes susceptibles de alta hospitalaria, ya sea sólo por alta a domicilio, o porque el paciente, dado su cuadro clínico, reciba el alta en condiciones de hospitalización a domicilio.

Para el diseño del plan de responsabilidad social corporativa, además de considerar los resultados de la investigación precedente, se ha contado con la participación y asesoramiento de un panel de expertos en los temas sobre los que versa el citado proyecto. Este equipo estaba formado por Belén Mercedes Urosa Sanz (decana de la Facultad de Ciencias Humanas y Sociales de la Universidad Pontificia de Comillas), María Teresa García Nieto (profesora titular de Universidad de la Facultad de Ciencias de la Información de la Universidad Complutense de Madrid), María Arántzazu Álvarez de Arcaya Vicente (coordinadora médico 
de la Unidad de Medicina Hospitalaria del Hospital Clínico San Carlos de Madrid) y Javier Echanove Ravello (director general y responsable de finanzas de la entidad no lucrativa 'Desarrollo y Asistencia. Solo en Madrid', que cuenta con 2000 voluntarios especializados en acompañamiento).

Entre los objetivos del plan de RSC, es preciso destacar los siguientes puntos. Entre los objetivos generales se pretendía, por un lado, humanizar la atención al paciente y mejorar su satisfacción, así como la de su familia y las personas de su entorno y, por otro, fomentar el sentimiento de pertenencia de los profesionales con relación a la organización, impulsando el compromiso con los valores éticos.

Entre los objetivos específicos en relación con cada grupo de público destacaban los siguientes puntos. En los objetivos en relación con los usuarios se buscaba:

- Mejorar la percepción de la atención hospitalaria en el paciente, sus familiares y su entorno.

- Mitigar el sentimiento de indefensión, soledad y abandono.

- Mejorar la confianza en la institución y los profesionales.

- Mejora de la calidad percibida, al sentir que se tienen en cuenta las necesidades individualizadas.

- Mejorar la eficiencia en la adecuación de la hospitalización, facilitando la vuelta al entorno familiar.

Respecto a los objetivos en relación con los profesionales, se perseguía:

- Reforzar los valores éticos de los profesionales.

- Fortalecer el sentimiento de pertenencia a la organización.

- Mejorar la relación médico-paciente.

- Mejorar el desarrollo del proceso asistencial.

- Facilitar el trayecto de la desvinculación laboral integrando al personal que no se encuentra en activo (profesionales jubilados).

En relación a los objetivos vinculados con los profesionales en formación se deseaba:

- Trasmitir los valores éticos a los profesionales que se están formando en la organización.

- Optimizar los programas de aprendizaje- servicio.

- Suscripción de convenios específicos con las universidades o escuelas profesionales para que, en determinados casos, la actividad realizada pueda ser reconocida como créditos por equivalencia o de libre elección. 
- Promover, con estas entidades académicas, la realización de actividades de información, asesoramiento y difusión de actividades de voluntariado de salud.

Finalmente, en los objetivos en relación con la organización, se buscaba

- Optimizar la eficiencia de los recursos (modelo de relaciones excelentes con los usuarios).

- Mejorar la notoriedad pública y la imagen social de la organización.

- Mejorar el prestigio de la institución.

- Crear valor compartido (que es la esencia de la RSC).

Respecto al desarrollo del plan de RSC, el desarrollo del proyecto se planteaba desde una estructura de responsabilidad social corporativa transversal a la organización, de manera que se garantizara su correcta y completa implementación. Los recursos tanto materiales como humanos dependen de la organización. Este procedimiento conlleva una serie de fases. La primera consiste en identificar la población objetiva o target audience. Se definirán las características de los pacientes que van a formar parte del programa de acompañamiento y los criterios de inclusión. Sus dificultades sociales deben concretarse en las carencias o en las insuficiencias de apoyo familiar y con necesidad de atención sanitaria. Serán pacientes con un nivel moderado de dependencia.

La segunda fase busca analizar la viabilidad y estrategias de aplicación. Pretende identificar los recursos necesarios tanto materiales como humanos. También quiere crear una estructura de coordinadores expertos y establecer planes de formación desde las mismas organizaciones implicadas. También pretende diseñar coberturas, horarios y cuadros de mando.

La tercera fase es el Plan de Acción o Medidas de Implementación. Consiste en establecer las acciones a llevar a cabo como el acompañamiento en la ambulancia, del hospital al domicilio del paciente, acompañamiento a revisiones médicas, consultas o residencias.

\section{Evaluación de resultados}

Con la evaluación se pretende medir el impacto que genera la plataforma implementada en los colectivos que participan en el proyecto. Para ello se establecen los procedimientos y sus indicadores de medición. El público prioritario es el de los usuarios, los pacientes. Para evaluar la eficacia de la plataforma en este colectivo se aplicarán encuestas de satisfacción del paciente y cuestionarios de calidad percibida con diferentes escalas.

Los siguientes beneficiarios son los profesionales. Se obtendrán indicadores mediante encuestas de clima laboral, evaluaciones de desempeño y el porcentaje de reclamaciones de trato. Se realizará un 
estudio pre y post de estos indicadores para poder comprobar la eficacia del plan. También son beneficiarios los profesionales en formación. Se obtendrán indicadores mediante cuestionarios de feedback, análisis del número de solicitudes para formarse en la organización (estudio pre y post) y encuestas a los centros de formación. Percepción sobre el número y el contenido de las charlas impartidas en los centros de formación colaboradores con la organización.

Respecto de la propia organización se realizarán encuestas de percepción social. Se emprenderá un análisis comparativo del tipo de noticias en comunicación interna y externa, antes y después de la implantación del proyecto, tanto de las remitidas, como de las publicadas. Además, se considerarán indicadores útiles las encuestas de satisfacción del paciente. Y se valorará el impacto de la memoria de responsabilidad social corporativa que incluya este plan.

\section{Conclusiones}

En las actuales sociedades occidentales, todo acto de atención sanitaria implica el intento científico de curar una enfermedad y el empeño de cuidar al paciente. En este sentido, la planificación de los servicios sanitarios, además de centrarse en optimización de recursos tangibles, debe orientarse al cultivo de la sensibilidad social y de los valores inherentes a la atención sanitaria. De este modo, y como consecuencia, la organización adquirirá prestigio y ganará autoridad moral frente al conjunto de la sociedad.

El camino hacia un nuevo modelo pasa por considerar las necesidades y expectativas de los pacientes, así como las actitudes y comportamientos de los profesionales. Es preciso que el profesional sanitario conozca, entienda y asuma el punto de vista del paciente. No podemos olvidar que es en los profesionales en los que recae gran parte de la responsabilidad de la percepción que tienen los pacientes sobre el servicio recibido.

La eficiencia económica y la efectividad sanitaria han sido los puntos fundamentales sobre los cuales se viene planteando la modernización del sistema sanitario. No obstante, se hace necesario añadir una nueva política basada en la orientación al paciente.

La implantación de una plataforma de voluntariado para acompañamiento al paciente al alta supone una serie de ventajas para todos los colectivos que están implicados. El principal beneficiario será el propio paciente quien, con el apoyo del voluntariado, verá satisfechas sus necesidades de acompañamiento, favoreciendo una percepción de la calidad sanitaria mucho más positiva.

Los segundos, y no por ello menos importantes, serán sus familiares y las personas de su entorno, quienes, dada la realidad actual, se enfrentan con dificultades para asumir el cuidado del paciente debido a las cargas personales y laborales. La plataforma les proporciona un respiro.

Los terceros beneficiarios serán los profesionales sanitarios, quienes pueden concentrarse en sus tareas propias durante su jornada laboral y, además, podrán desarrollar otras actividades complementarias 
formando parte de la plataforma de acompañamiento. Nos referimos a trabajadores en activo, jubilados, residentes y otros profesionales en formación. Se trata de crear y fomentar una cultura de valores, y de reforzar el sentimiento de pertenencia a la organización.

Junto a todo lo anterior, con la implementación de este proyecto de RSC, contribuimos a que la organización hospitalaria resulte más visible para los ciudadanos y tenga una imagen más integrada en la sociedad, como una organización más sensible y comprometida con la responsabilidad social corporativa.

Sin embargo, el mayor beneficiario del desarrollo del presente proyecto será, sin duda, el sistema sanitario español. Agilizar las altas proporcionará una mejora de la calidad sanitaria percibida en los ciudadanos y, al tiempo, supondrá una reducción de los costes propiciando, así, la optimización de los recursos públicos.

\section{Referencias}

- $\quad$ Advice P.R.N. training volunteers: Watch your step. (2005). Nursing, 35(9), 14-14.

- $\quad$ Carabaña Morales, J. (2003). Los cambios demográficos y sus consecuencias sociales. Información Comercial Española, ICE: Revista de Economía, 811, 153-174.

- $\quad$ Carroll, A.B. (1991). The Pyramid of Social Responsibility: Toward the Moral Management of Organizational Stakeholders. Business Horizons, july-august, 39-48.

- $\quad$ Carroll, A.B. (1999). Corporate social responsibility: evolution of a definitional construct. Business and Society, vol.38, n 3, 268-295.

- $\quad$ Carroll, A.B. y Buchholtz, A.K. (2011). Business and Society: Ethics, Sustainability, and Stakeholder Management, 8th Edition. Boston: South-Western College Publishing.

- $\quad$ Dozier, D. M., with Grunig, L. A., \& Grunig, J. E. (1995). Manager's guide to excellence in public relations and communication management. Mahwah, $\mathrm{NJ}$ : Lawrence Erlbaum.

- $\quad$ Freeman, R. E. (1983). Stockholders and Stakeholders: A New Perspective on Corporate Governance. California Management Review; Spring 25, 3; pg. 88-106.

- $\quad$ Freeman, R. E. y Gilbert, D. R. (1988). Corporate Strategy and the Search for Ethics. Englewood Cliffs. NJ: Prentice Hall.

- $\quad$ Freeman, R.E.; Wicks, A.C. y Parmar, B. (2004). Stakeholder Theory and "The Corporate Objective Revisited" Organización Science 15 (3), pp 364-369.

- $\quad$ Fundació Víctor Grífols i Lucas (2005). Los fines de la medicina - Els fins de la medicina. Cuadernos de la Fundació Víctor Grífols i Lucas n¹1. Barcelona.

- $\quad$ FUNDADEPS. Fundación de Educación para la Salud (2011). El Aula Social Dr Zarco, un compromiso del Hospital Clínico con la sociedad. http://www.adeps.org/EpsOpiniones.asp?codopinion=46. Consultado 14-2016.

- $\quad$ García Nieto, M.T. (2012). Las Ciencias Sociales y la Responsabilidad Social Corporativa. aDReseach. ESIC.Vol 6 julio-diciembre, pp 92-111.

- $\quad$ Grunig, J.E. \& Hunt, T. (1984): Managing Public Relations. New York: Holt, Rinehart and Winston.

- $\quad$ Grunig, J. E. (ed.) (1992). Excellence in public relations and communication management. Hillsdale, NJ: Lawrence Erlbaum. 
- $\quad$ Grunig, L. A., Grunig, J. E., \& Vercic, D. (1998). Are the IABC's excellence principles generic? Comparing Slovenia and the United States, the United Kingdom and Canada. Journal of Communication Management., 2, 335-356.

- $\quad$ Grunig, L. A., Grunig, J. E., \& Dozier, D. M. (2002). Excellent public relations and effective organizations: A study of communication management in three countries. Mahwah, NJ: Lawrence Erlbaum.

- $\quad$ Grunig, J. E. (2006). Furnishing the edifice: Ongoing research on public relations as a strategic management function. Journal of Public Relations Research, 18, 151-176.

- $\quad$ Grupo Innova-Docencia 281 de la UCM (2017). Universidad y sociedad: comunicación e integración en empresas e instituciones públicas y organizaciones no lucrativas. En Rodríguez Torres, Javier: Retos docentes como desafío curricular. Madrid: McGrawHill.

- Instituto Nacional de Estadística (2015). España en cifras. Madrid: INE.

- $\quad$ Mendoza Giraldo, D., Navarro, A., Sánchez-Quijano, A., Villegas, A., Asencio, R., \& Lissen, E. (2012). Impact of delayed discharge for nonmedical reasons in a tertiary hospital internal medicine department. Revista Clínica Española, 212(5), 229-234.

- $\quad$ Rydeman, I., Törnkvist, L., Agreus, L., \& Dahlberg, K. (2012). Being in-between and lost in the discharge process. An excursus of two empirical studies of older persons', their relatives', and care professionals' experience. International Journal of Qualitative Studies on Health \& Well-being, 7, 1-9. doi:10.3402/qhw.v7i0.19678.

- $\quad$ Toth, E. L. (ed.) (2007). The Future of Excellence in Public Relations and Communication Management: Challenges for the Next Generation. London: Lawrence Erlbaum Associates.

- Wolfe, L. M. (2013). Hospital readmissions: The need for a coordinated transitional care model: Analysis and synthesis of research on medicare policy and interventions for the elderly. Dissertation Abstracts International Section A, 74. 